\title{
Sensitivity analysis on asymmetric stud anchor chain
}

\author{
Cunjun $\mathrm{LI}^{1}$, Xinyue $\mathrm{LI}^{2}$, Zuhua JIANG ${ }^{2}$ \\ ${ }^{1}$ Zhoushan Institute of Calibration and Testing for Quality and Technology Supervision, Zhejiang, \\ China \\ ${ }^{2}$ Shanghai Jiao Tong University, Shanghai, China
}

Keywords: Sensitivity analysis, stud anchor chain, finite element method (FEM)

\begin{abstract}
Caused by the manufacturing process and equipment, dimensional deviations occur in the anchor chain and lead to asymmetry. In order to evaluate the stress in asymmetric chain, this paper uses finite element method (FEM) to model the $76 \mathrm{~mm}$ asymmetric stud anchor chain and calculate the stress distribution under the specific load. Simulating different scales of deviation, this paper presents the sensitivity analysis on the asymmetry. From the FEM results, when the central point moves upward, the stress in the bottom waist is increased, but the variation is not obvious; the stress in the upper waist is decreased, and the amplitude is large. The different between the bottom and upper waist is increased.
\end{abstract}

\section{Introduction}

Anchor chain, which is a chain linking the anchor and the hull, passes and buffers the external force on the ship, and also generates part of friction force. Caused by the manufacturing process and equipment, dimensional deviations occur in the anchor chain, hence called Asymmetry of manufacturing. Asymmetry is usually performed as the deviation of central point, when the width of anchor chain (called height in the following paper, corresponding to the FEM model) stays the same. This situation will trigger the change of the force-bearing point and the stress on each part of the chain body, which directly influence the life and quality of the anchor chain.

Scholars designed and utilized many approaches to calculate the distribution of stress in common stud anchor chain. Formulas were proposed to evaluate the maximum stress in the chain body, based on the current design and not included shape parameters [1-3]. Japanese scholar assumed the strain in the connection area of the chain as 0 , and then calculated the influence on stress in chain body under the situations of different size of connection area [4].

This paper chooses $76 \mathrm{~mm}$ asymmetric stud anchor chain, and presents the sensitivity analysis on the asymmetry.

\section{FEM model and calculation}

The process of FEM is as following: First, the solid model of the chain is constructed, which contains some degree of dimensional asymmetry; then, the chain is loaded by the connection of another link. Considering the symmetry of the model, $1 / 4$ of the model is selected in the FEM calculation.

For FEM model of $76 \mathrm{~mm}$ anchor chain, load is $4731 \mathrm{kN}$, Young modulus of the chain is 2.07e11Pa, Poisson ratio is 0.3 , and friction coefficient is 0.05 . Length and height (width) of the chain is fixed, while the location of central point is variated horizontally. In FEM calculation, since the chain is up-down symmetric and the results are the same when the central point deviates symmetrically, this paper only consider the situations of upward deviation. The scale of deviation is $1 \%, 2 \%, 3 \%, 4 \%$ and $5 \%$. Namely, if the scale is set to $1 \%$ and the height of chain is $1.6 \mathrm{D}$, then the deviation is $1.6 \mathrm{D}^{*} 1 \%=0.016 \mathrm{D}$ and the central point shift upward $0.016 \mathrm{D}$. Sensitivity analysis is focus on the influence on stress distribution under different cases of dimensional variation. 


\section{Sensitivity analysis on $\mathbf{7 6} \mathrm{mm}$ asymmetric stud anchor chain}

$76 \mathrm{~mm}$ asymmetric stud anchor chain (length is 6D and width is 1.6D) is concentrated in FEM process. Following results of 1\% 5\% scale of deviation are obtained:

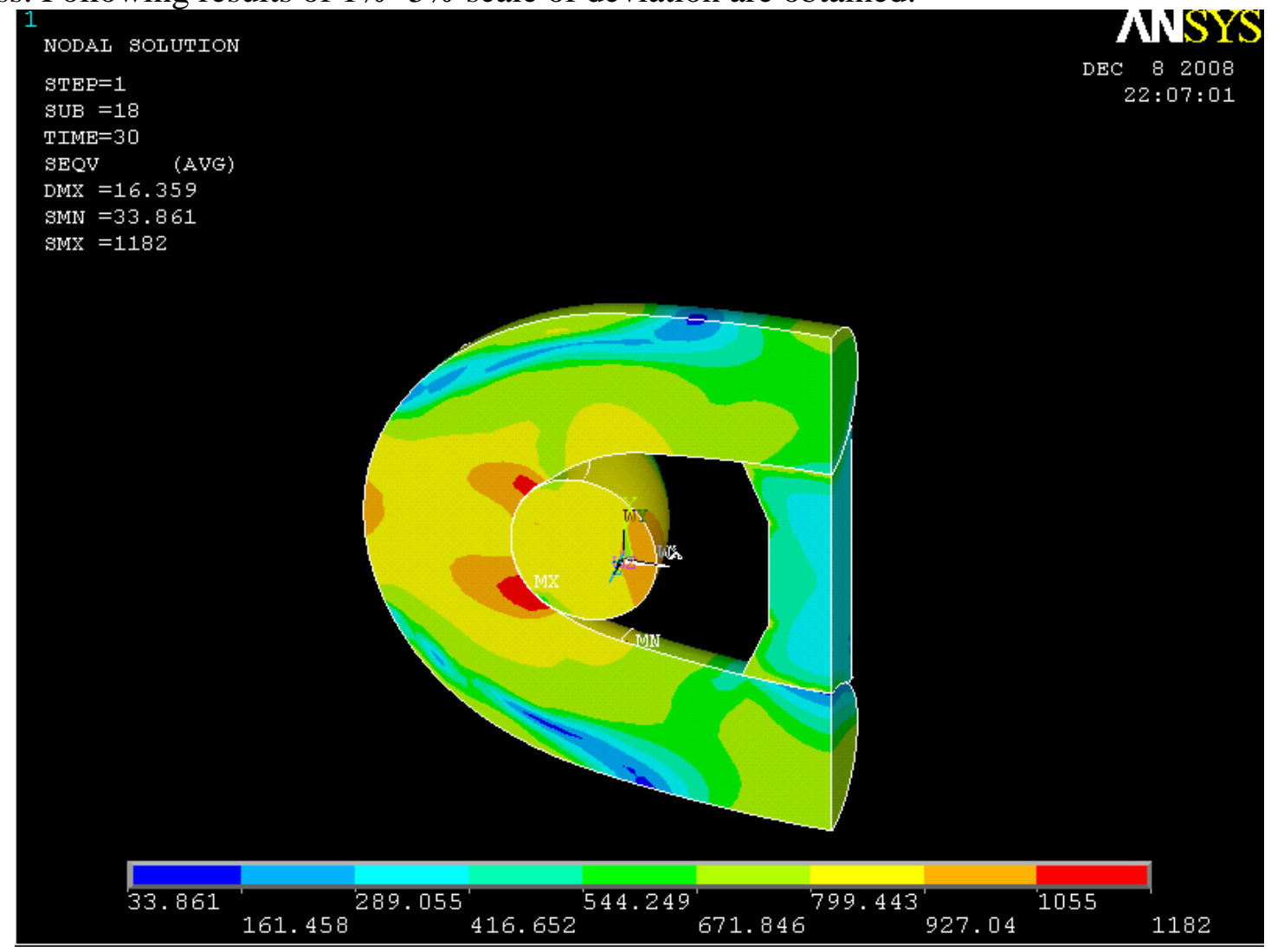

Fig.1. Stress distribution of $76 \mathrm{~mm}$ anchor chain, $5 \%$ deviation

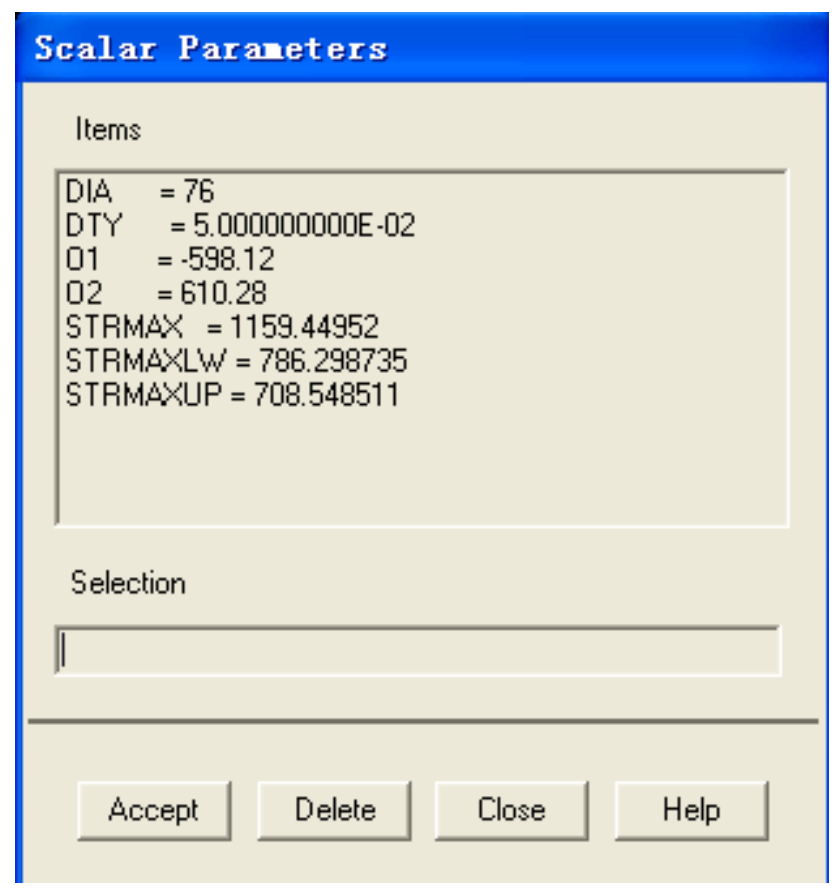

Fig.2. Scalar parameters of $76 \mathrm{~mm}$ anchor chain, $5 \%$ deviation

From figure 1 and 2, the minimum stress in anchor chain locates in the both ends of the external arcs (blue-colored area), while the maximum stress locates in the peak of internal arcs (red-colored area). Caused by the asymmetry, red-colored area is divided into two parts.

In all 5 cases, studs carry some load, but the stresses are not too large (300 400MPa). 
This paper also calculates the stress in the welded junction of the waist.

Table 1 . Stresses in 5 cases of $76 \mathrm{~mm}$ anchor chain

\begin{tabular}{ccccc}
\hline Scale & Max Stress & Stress in bottom waist & Stress in upper waist & Difference \\
\hline $1 \%$ & 1158 & 783 & 781 & 2 \\
$2 \%$ & 1159 & 783 & 780 & 3 \\
$3 \%$ & 1153 & 784 & 762 & 22 \\
$4 \%$ & 1160 & 785 & 734 & 51 \\
$5 \%$ & 1159 & 786 & 709 & 77 \\
\hline
\end{tabular}

The maximum stresses in 5 cases are not over the allowed range, and the line chart is presented.

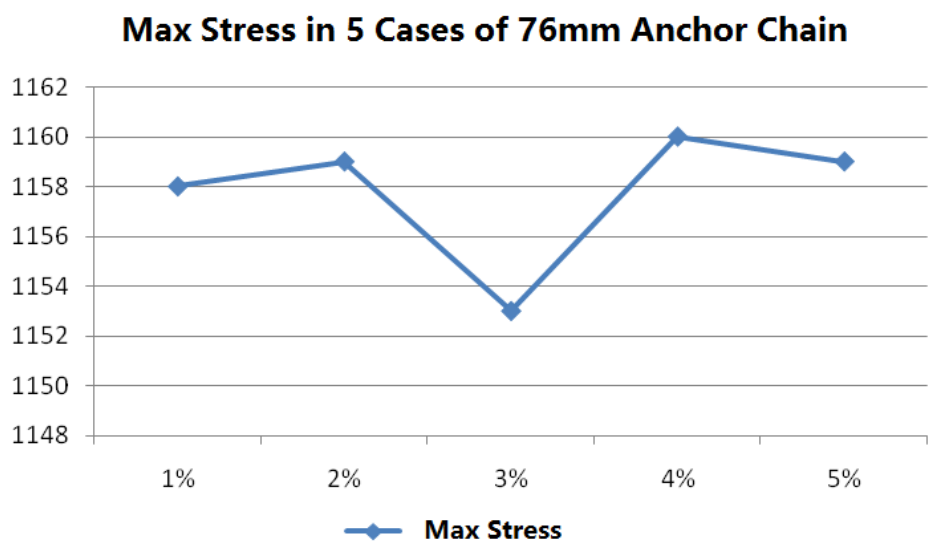

Fig.3. Max stress of $76 \mathrm{~mm}$ anchor chain

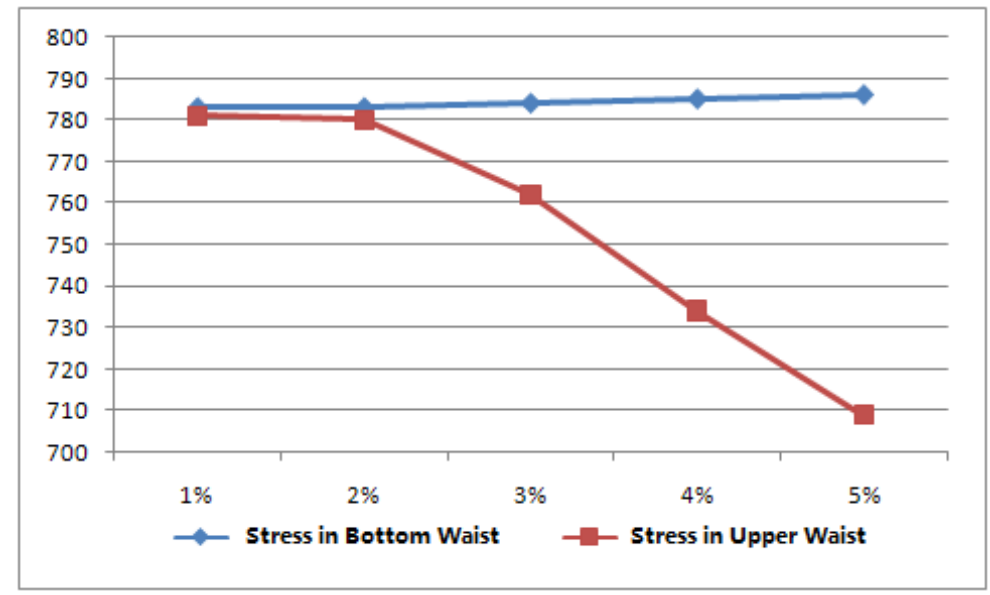

Fig.4. Stress in the bottom and upper waist

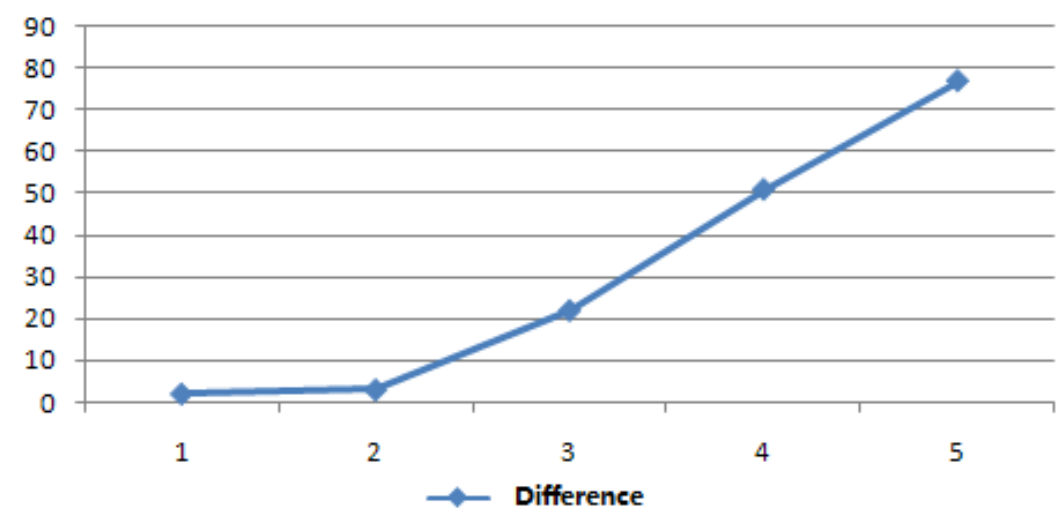

Fig.5. Difference between the bottom and upper waist

From figure 3, when the central point moves upward, the maximum stress is not changed with a certain rule. An abrupt change happened when the scale enlarged to $3 \%$, and the minimum value 
among 5 cases is achieved. When scale is set to 4\%, chain carries maximum load $1160 \mathrm{MPa}$.

From figure 4, when the central point moves upward, the stress in the bottom waist is increased, but the variation is not obvious; the stress in the upper waist is decreased, and the amplitude is large.

From figure 5, when the central point moves upward, the different between the bottom and upper waist is increased. It's the upward deviation of central point that causes the phenomenon.

\section{Conclusion}

Considering the upward deviation of central point ranging from $1 \%$ to $5 \%$, this paper presents a series of research on $76 \mathrm{~mm}$ asymmetric stud anchor chain.

Using line graph, this paper visualizes the influence of stress under different deviation of central point. Not only the maximum stress, but also the stresses in bottom and upper waist are considered in this paper. Empirical influence of difference between the bottom and upper waist caused by the upward deviation is then concluded.

For $76 \mathrm{~mm}$ asymmetric stud anchor chain, when the central point moves upward, the stress in the bottom waist is increased, but the variation is not obvious; the stress in the upper waist is decreased, and the amplitude is large. The different between the bottom and upper waist is increased.

\section{References}

[1] Papazoglou V I, Katsaounis G M, Papaioannou J D. Elastic static analysis of chain links in tension and bending[J]. International Journal of Offshore and Polar Engineering, 1991, 1(04).

[2] Lassen T, Syvertsen K. Fatigue Relaibility And Life-Cycle Cost Analysis of Mooring Chains[J]. International Journal of Offshore and Polar Engineering, 1997, 7(02).

[3] Hobbs R E, Ridge I M L. Torque in mooring chain. Part 1: background and theory[J]. The Journal of Strain Analysis for Engineering Design, 2005, 40(7): 703-713.

[4] Suzuki N, Soya I, Nitta H, et al. Effect of the link shape of large chain on stress distribution[J]. Tetsu-to-Hagane(J. Iron Steel Inst. Jpn.), 1987, 73(16): 2275-2281. 